\title{
AVALIAÇÃO DA COORDENAÇÃO MOTORA EM CRIANÇAS E ADOLESCENTES COM DEFICIÊNCIA AUDITIVA: UMA REVISÃO SISTEMÁTICA DE ESTUDOS BRASILEIROS
}

\author{
Marília Silva Guedes, Universidade do Estado da Bahia - UNEB, Bahia - Brasil \\ Jorge Lopes Cavalcante Neto, Universidade do Estado da Bahia-UNEB, Bahia-Brasil
}

\section{RESUMO}

O objetivo do estudo foi analisar por meio de revisão sistemática informações acerca das formas de avaliação da coordenação corporal em crianças e adolescentes com deficiência auditiva em estudos brasileiros. Para tanto, utilizaram-se os seguintes descritores: Coordenação Motora, Testes, Surdez e foram feitas combinações dos mesmos com o indicador booleano AND. As buscas foram realizadas nas seguintes bases de dados: Periódicos CAPES, Scielo, Lilacs e Google Scholar. Foram selecionados 05 artigos por obedecerem aos critérios de inclusão. Observou-se que o público alvo foram crianças e adolescentes de 07 a 18 anos de ambos os sexos nas regiões Sul e Sudeste, encontrando poucos artigos referentes à temática. Houve uma preocupação pelos autores em detalhar os instrumentos para coleta de dados, sendo o KTK o teste mais utilizado para avaliar a coordenação motora de crianças com deficiência, composta por quatro tarefas: trave de equilíbrio, salto monopedal, salto lateral e transferência sobre a plataforma, o qual ficou constatado que após um período de intervenção com aulas de Educação Física as crianças tiveram resultado significantes na realização dos testes. A limitação dos estudos foi referente à amostra ter sido pequena e pelo período de intervenção ser curto. Por fim, os autores apontaram a importância de um programa de Educação Física estruturado para aquisição da coordenação motora de criança com deficiência auditiva, tendo um período de intervenção específica para haver melhoras nas habilidades motoras.

Palavras-Chave: Coordenação motora; Testes; Surdez.

\section{EVALUATION OF MOTOR COORDINATION IN CHILDREN AND ADOLESCENTS WITH HEARING LOSS: A SYSTEMATIC REVIEW OF BRAZILIAN STUDIES}

\begin{abstract}
The aim of this study was to analyze by means of systematic review information about the forms of evaluation of body coordination in children and adolescents with hearing loss in Brazilian studies. To this end, the following descriptors were used: Motor skills, Tests, Deafness and combinations thereof have been made with the boolean indicator AND. The searches were conducted in the following databases: Periódicos CAPES, Scielo, Lilacs and Google Scholar. 05 articles were selected because they satisfy the criteria for inclusion. It


was observed that the target audience were children and adolescents from 07 to 18 years for both sexes in the South and Southeast, finding a few articles related to the subject. There was a concern for authors in detail the data collection instruments, being the most used test for KTK assess motor skills of children with disabilities, consisting of four tasks: balance beam, vault, side and heel monopedal transfer on the platform, which was established after a period of Physical education classes with children had significant results in the performance of the tests. The limitation of the studies was referring to the sample have been small and the period of intervention be short. Finally, the authors pointed out the importance of a structured Physical education program for acquisition of motor skills of children with hearing impairment, having a specific period to be improvements in motor skills.

Key-Words: Motor skills; Tests; Deafness.

\section{EVALUACIÓN DE LA COORDINACIÓN MOTRIZ EN NIÑOS Y ADOLESCENTES CON PÉRDIDA AUDITIVA: UNA REVISIÓN SISTEMÁTICA DE ESTUDIOS BRASILEÑOS}

\section{RESUMEN}

El objetivo de este estudio fue analizar por medio de la revisión sistemática de información sobre las formas de evaluación de la coordinación del cuerpo en niños y adolescentes con pérdida de la audición en los estudios brasileños. Para ello fueron utilizados los siguientes descriptores: motricidad, pruebas, sordera y combinaciones de las mismas han sido realizadas con el indicador booleano y. Se realizaron búsquedas en las bases de datos: Revistas CAPES, Scielo, Lilacs y Google Scholar. 05 artículos fueron seleccionados porque cumplen los criterios de inclusión. Se observó que el público objetivo fueron los niños y adolescentes de 07 a 18 años para ambos sexos en el sur y sudeste, encontrando un par de artículos relacionados al tema. Hubo una preocupación por autores en detalle los instrumentos de recolección de datos, siendo la prueba más usada para KTK evaluar habilidades motoras de los niños con discapacidades, que consta de cuatro tareas: transferencia monopedal viga de balance, bóveda, laterales y del talón de la plataforma, que se estableció después de un período de clases de educación física con los niños tenía resultados significativos en la realización de las pruebas. La limitación de los estudios se refiere a la muestra han sido pequeños y el período de intervención ser corto. Por último, los autores señalaron la importancia de un programa estructurado de educación física para la adquisición de las habilidades motoras de los niños con discapacidad auditiva, teniendo un período específico que mejoras en las habilidades motoras.

Palabras-Clave: Coordinación motora; Pruebas; Sordera. 


\section{INTRODUÇÃO}

A deficiência auditiva pode ser caracterizada como uma perda total ou parcial da capacidade de ouvir ou perceber sinais sonoros. ${ }^{1}$ E por não ser um problema sensorial visível, pode trazer problemas sérios para o individuo se não for diagnosticado nos primeiros anos de vida, porque o diagnóstico tardio pode levar a implicações cognitivas, linguísticas e emocionais. ${ }^{2}$

A perda auditiva pode ocorrer em três diferentes regiões, sendo denominadas lesões condutivas, nas quais o individuo apresenta diminuição na intensidade sonora, lesões neurosensoriais, na parte interna do ouvido, que caracteriza perdas na intensidade e percepções do som e ainda lesões centrais, que atingem a partir do tronco cerebral até as regiões subcorticais e o córtex. ${ }^{1,3}$

Sendo assim, as crianças que apresentam sinais de perda auditiva devem ser avaliadas o mais rápido possível, pois, muitas vezes a perda auditiva na infância só é percebida numa faixa etária avançada, o que dificulta a tomada de medidas cabíveis para uma estimulação adequada. Sendo que na maioria dos casos o problema auditivo é observado pelos familiares quando a criança não responde a alguns sinais sonoros e também pela ausência da fala, dificultando assim o desenvolvimento da comunicação e linguagem desta. ${ }^{4}$

Alguns estudos ${ }^{5-6}$ afirmam que a pessoa com perda auditiva pode apresentar distúrbios na fala, no desenvolvimento motor, emocional e social, afetando assim a realização de atividades diárias, pois, como a audição é um meio de comunicação fundamental para a aquisição da fala, a perda pode levar muitos indivíduos ao isolamento social, além de comprometer na coordenação motora, equilíbrio e ritmo, dificultando na aprendizagem e atenção. $^{7}$

Esses comprometimentos motores implicam no inicio da infância, quando a criança está desenvolvendo suas habilidades motoras fundamentais, como andar, correr, saltar, arremessar entre outros. ${ }^{8}$ Pois uma criança quando recebe estímulos motores desde cedo é capaz de ter melhor controle no equilíbrio e coordenação, entretanto uma criança com surdez se não tiver estímulos motores desde cedo pode ter maiores comprometimentos, uma vez que o desempenho motor na condição de deficiência é mais complexo. ${ }^{6}$ 
Pellegrini et al. ${ }^{9}$ também diz que a coordenação motora é fundamental para a produção do movimento, pois vários músculos são ativados fazendo com que o individuo tenham maior amplitude e velocidade e dependendo da complexidade do movimento e/ou tarefa o individuo precisará de maiores habilidades motoras.

Sendo assim, estudiosos criaram alguns testes para avaliar a coordenação motora e equilíbrio de crianças com deficiência, dentre eles o KTK desenvolvido pelos pesquisadores alemães Kiphard e Schilling, ${ }^{10}$ construído para diagnosticar deficiências motoras em crianças com lesões cerebrais e/ou desvios comportamentais, ${ }^{11}$ sendo composto de atividades para avaliar o equilíbrio, ritmo, lateralidade, velocidade e agilidade.

O equilíbrio é caracterizado como a manutenção de forças que tem o objetivo de sustentar o corpo sobre uma base contra a gravidade, sendo assim, observa-se que o equilíbrio é exigido na primeira tarefa do KTK, que visa avaliar o equilíbrio dinâmico. ${ }^{12} \mathrm{E}$ tratando-se de pessoas com deficiência auditiva estudos apontam ${ }^{13-15}$ que boa parte desses indivíduos pode apresentar comprometimento no equilíbrio, uma vez que, o aparelho vestibular, que é responsável pelo equilíbrio, pode ser afetado, influenciando no desempenho motor do individuo.

Esse comprometimento no equilíbrio também pode interferir em outras valências físicas do individuo com surdez, como o ritmo, definido como encadeamento de tempo e movimento, caracterizado por repetições periódicas. ${ }^{12}$ Assim como a lateralidade, ${ }^{16}$ que, relaciona-se ao esquema interno do individuo em possuir maior facilidade motora de um lado do corpo em à relação ao outro, como observado na tarefa de transferência sobre a plataforma, em que há o deslocamento do indivíduo sobre as mesmas em um determinado tempo. ${ }^{11}$

Já a velocidade, que é caracterizada pela rapidez de execução de uma contração muscular no curto espaço de tempo, ${ }^{12}$ pode ser observada na tarefa de saltos laterais, ${ }^{11}$ em que o individuo tem que realizar o maior número de saltos em um determinado tempo. Além da velocidade, essa tarefa trabalha a agilidade, que é definida como a capacidade de mudar a posição do corpo ou direção em um curto período de tempo. ${ }^{17}$ 
Associando a bateria de teste KTK juntamente com intervenções com aulas de Educação Física, diversos autores ${ }^{6-8,18}$ apontaram que a coordenação motora de crianças com deficiência auditiva apresentou comprometimento, dificultando na realização do teste KTK. Contudo, tiveram melhora na coordenação motora após as aulas de Educação Física, pois, foram vivenciadas atividades que estimulassem o desenvolvimento motor das crianças, mostrando a importância da atividade física na vida desses indivíduos para aquisição de habilidades motoras.

Silva ${ }^{19}$ na sua pesquisa analisou o nível de desenvolvimento motor de crianças surdas que praticavam natação escolar, e constatou que as crianças tiveram ganhos significativos na respiração e equilíbrio, e que o papel do professor de Educação Física com atividades estruturadas, jogos e brincadeiras foi fundamental para o desenvolvimento global da criança surda, além de aprimorar as esferas cognitiva, motora e auditiva. ${ }^{20}$

Sabendo-se da importância da atividade física para aquisição da coordenação motora de crianças e adolescentes com deficiência auditiva, e devido à escassez de estudos nacionais que sistematizem a produção do conhecimento sobre a avaliação motora nessa população, o objetivo do presente estudo foi analisar por meio de uma revisão sistemática informações acerca das formas de avaliação da coordenação corporal em jovens com deficiência auditiva em estudos brasileiros.

\section{MÉTODOS}

Trata-se de uma revisão sistemática acerca das formas de avaliação da coordenação motora de jovens com deficiência auditiva realizada no período de 10 de dezembro de 2013 a 12 de março de 2014, utilizando-se dos seguintes descritores em português: Coordenação Motora, Testes, Surdez. Os mesmos foram retirados da base de Descritores em Ciências da Saúde (DECS) da Biblioteca Virtual de Saúde (BVS), sendo feitas combinações dos mesmos com o auxílio do indicador booleano AND.

A pesquisa foi realizada nas seguintes bases de dados: Periódicos CAPES, Scielo, Lilacs e Google Scholar. Recorreu-se a tais bases por elas agregarem as maiores produções 
oriundas da América Latina e consequentemente brasileiras. Devido o objetivo do estudo de somente revisar produções nacionais, não foram usados descritores em língua inglesa, nem base de dados com maior concentração de periódicos internacionais.

Apesar do elevado impacto que as produções internacionais apresentam, faz-se necessário listar e discutir as publicações em língua portuguesa oriundas de pesquisadores do Brasil, visando disseminar nacionalmente, no campo da Educação Física e Ciências do Esporte, as implicações dessa temática de estudo, ainda tão limitada no país.

Como critérios de elegibilidade para a presente revisão foram estabelecidos: artigos em língua portuguesa, publicados nos últimos 10 anos, que fossem de acesso livre e que tivessem como objetivo a avaliação/intervenção da coordenação motora de crianças e/ou adolescentes com deficiência auditiva.

A busca bibliográfica realizada nessas bases de dados permitiu a localização de 9 artigos no total, sendo 4 artigos na Revista Brasileira de Educação Especial, 2 na Revista CEFAC, 1 artigo na Revista Brasileira de Crescimento e Desenvolvimento Humano, 1 artigo na Revista da Educação Física /UEM e 1 artigo na Revista Motricidade.

Além da busca realizada nas bases de dados mencionadas, foram feitas pesquisas em periódicos de referência no campo da motricidade humana, que não estavam indexados nas bases de dados. Assim, recorreu-se às revistas científicas: Sendo 2 artigos na revista Conexões da UNICAMP, 1 artigo na Coleção Pesquisa em Educação Física e por fim 1 artigo na revistas UNIPAR, encontrando 4 artigos de interesse.

Os resultados destas análises foram subdivididos em etapas, ou seja, inicialmente foram feitos a leitura dos títulos, depois a leitura dos resumos e por fim a leitura dos textos completos. Sendo que um dos critérios de inclusão deste estudo é que fossem artigos com resultados de pesquisa de campo e que no titulo e/ou resumo tivesse alguma das palavraschave ou sinônimos. 
Inicialmente, localizou-se um total de 13 artigos, os quais foram analisados pelo título, após a leitura do resumo 7 artigos foram excluídos, sendo que 2 destes eram de revisão bibliográfica, não obedecendo ao critério de inclusão do estudo. 6 artigos foram selecionados para análise detalhada por meio da leitura dos textos completos, e destes 6 artigos, 1 foi excluído por não deixar esclarecidos os métodos e instrumentos da pesquisa. Por fim, 5 artigos foram analisados na integra em relação aos objetivos, métodos e instrumentos de pesquisa e os principais achados. O processo de seleção dos textos pode ser visualizado na Figura 1:

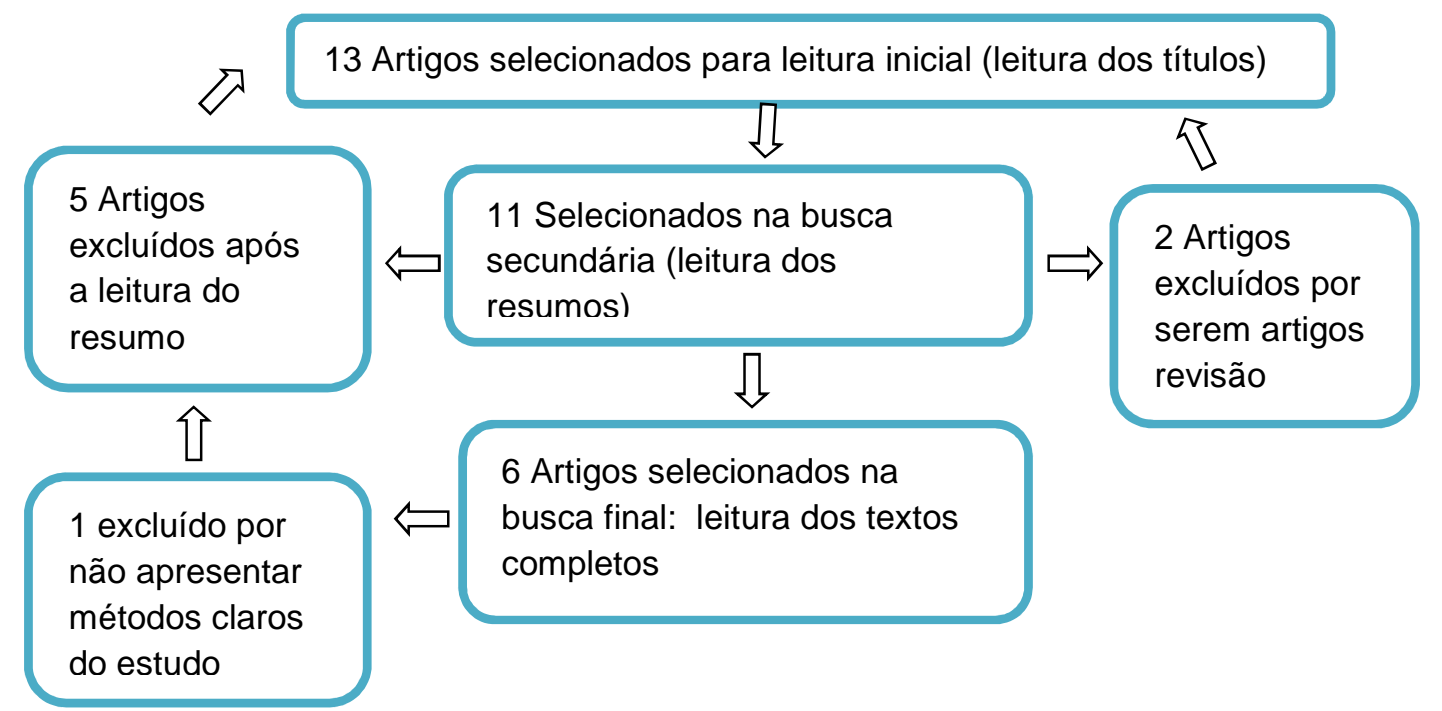

Figura 1: Organograma das etapas de seleção dos artigos.

\section{RESULTADOS E DISCUSSÃO}

Verificou-se que os 05 artigos selecionados nesta revisão foram publicados no período de 2008 até 2011. Observou-se certa concentração geográfica na região Sudeste com 03 trabalhos publicados e na região Sul com a publicação de 02 trabalhos. 
Quadro 1 - Caracterização dos estudos selecionados para a presente Revisão Sistemática $(\mathrm{N}=05)$

\begin{tabular}{|c|c|c|c|c|c|}
\hline AUTORES & ANO & TITULO & LOCAL & OBJETIVOS & MÉTODOS \\
\hline $\begin{array}{l}\text { STRAPASSON } \\
\text { et al. }\end{array}$ & 2009 & $\begin{array}{l}\text { O teste ktk na } \\
\text { avaliação da } \\
\text { coordenação } \\
\text { motora de } \\
\text { pessoas com } \\
\text { deficiência } \\
\text { auditiva }\end{array}$ & Toledo-PR & $\begin{array}{l}\text { Avaliar a coordenação motora } \\
\text { dos alunos com deficiência } \\
\text { auditiva da apada; aplicar } \\
\text { aulas de educação física com } \\
\text { o intuito de ajudar no } \\
\text { desenvolvimento e/ou } \\
\text { melhora do item mencionado. }\end{array}$ & $\begin{array}{l}\text { Utilizou-se o teste de } \\
\text { coordenação } \quad \text { motora } \\
\text { ktk.Amostra composta por } \\
8 \text { alunos de ambos o sexos, } \\
\text { com idade entre } 8 \text { e } 13 \\
\text { anos. }\end{array}$ \\
\hline SOUZA et al. & 2008 & $\begin{array}{l}\text { Análise da } \\
\text { coordenação } \\
\text { motora de } \\
\text { pessoas surdas }\end{array}$ & Campinas- SP & 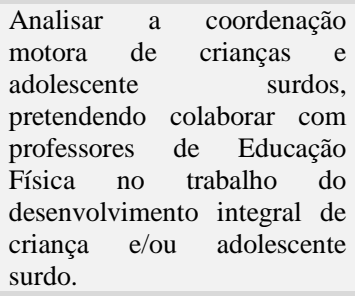 & $\begin{array}{l}\text { Utilizou-se o Teste de } \\
\text { Coordenação Corporal } \\
\text { KTK. Amostra foi de } 23 \\
\text { sujeitos surdos de ambos os } \\
\text { sexos, com idade de } 7 \text { a } 14 \\
\text { anos. }\end{array}$ \\
\hline $\begin{array}{l}\text { MONTEZUMA } \\
\text { et al. }\end{array}$ & 2011 & $\begin{array}{l}\text { Adolescentes } \\
\text { com deficiência } \\
\text { auditiva: a } \\
\text { aprendizagem } \\
\text { da dança e a } \\
\text { coordenação } \\
\text { motora }\end{array}$ & Londrina-PR & $\begin{array}{l}\text { Verificar a ocorrência de } \\
\text { modificações da coordenação } \\
\text { motora, atenção, participação, } \\
\text { interação, autoestima e } \\
\text { compreensão em adolescentes } \\
\text { com deficiência auditiva, } \\
\text { após a realização de aulas de } \\
\text { dança do tipo jazz dance. }\end{array}$ & $\begin{array}{l}\text { Utilizou-se o teste de } \\
\text { coordenação corporal ktk. } \\
\text { Amostra de } 5 \text { sujeitos com } \\
\text { surdez adquirida e } \\
\text { congênita, do gênero } \\
\text { feminino, com idade entre } \\
13 \text { e } 18 \text { anos. }\end{array}$ \\
\hline EVEDO et al. & 2009 & $\begin{array}{l}\text { Estudo } \\
\text { comparativo do } \\
\text { equilíbrio de } \\
\text { crianças surdas } \\
\text { e ouvintes }\end{array}$ & São Paulo-SP & $\begin{array}{l}\text { Comparar o equilíbrio } \\
\text { estático, dinâmico e } \\
\text { recuperado de crianças surdas } \\
\text { e ouvintes. }\end{array}$ & $\begin{array}{l}\text { Foram utilizados o teste } \\
\text { "do quatro" e de Romberg } \\
\text { (equilíbrio estático), teste } \\
\text { "passeio na trave" e de } \\
\text { Unterberger (equilíbrio } \\
\text { dinâmico), e giro de } 180^{\circ} \\
\text { (equilíbrio recuperado). } \\
\text { Foram avaliadas } 9 \text { crianças } \\
\text { surdas e } 18 \text { crianças } \\
\text { ouvintes, de } 9 \text { a } 12 \text { anos, de } \\
\text { ambos os sexos, em } 2 \\
\text { escolas municipais em SP }\end{array}$ \\
\hline PENA; GORLA & 2010 & $\begin{array}{l}\text { Coordenação } \\
\text { motora em } \\
\text { crianças com } \\
\text { deficiência } \\
\text { auditiva: } \\
\begin{array}{l}\text { avaliação } \\
\text { intervenção }\end{array}\end{array}$ & $\begin{array}{l}\text { Campinas- } \\
\text { SP }\end{array}$ & $\begin{array}{l}\text { Avaliar nessas crianças, } \\
\text { através da bateria de testes } \\
\text { ktk, seu nível de coordenação } \\
\text { motora. } \\
\text { Analisar a influência de uma } \\
\text { intervenção pedagógica, com } \\
\text { aulas de iniciação ao } \\
\text { basquetebol. }\end{array}$ & $\begin{array}{l}\text { Utilizou-se o teste de } \\
\text { coordenação corporal ktk. } \\
\text { participaram do estudo } 11 \\
\text { crianças, de ambos os sexos } \\
\text { com idade entre } 8 \text { e } 11 \\
\text { anos. }\end{array}$ \\
\hline
\end{tabular}

Através do Quadro 1 é possível visualizar as principais informações resumidas dos artigos selecionados e analisados na presente revisão.

Observou-se que o público alvo dos 05 artigos foi composto por crianças e adolescentes de 07 a 18 anos, de ambos o sexos com exceção de 01 estudo que foi realizado somente com gênero feminino com diagnóstico de surdez congênita ou adquirida, ${ }^{8} 01$ estudo realizado com crianças com perda auditiva neurosensorial profunda congênita e adquirida e com

Conexões: revista da Faculdade de Educação Física da UNICAMP, Campinas, v. 13, n. 3, p. 114-130, jul./set. 2015. ISSN: 1983-9030 
crianças ouvintes visando fazer a comparação, ${ }^{13} 02$ artigos foram feitos em um Instituto de ensino para crianças com deficiência auditiva, de Campinas - SP ${ }^{6-7}$ e por fim 01 estudo foi feito em uma associação que atende crianças com deficiência auditiva da cidade de Toledo, PR. ${ }^{18}$

A partir dos achados desses artigos observa-se que a maioria dos autores procurou fazer suas pesquisas em instituições e/ou associações, pois é um método, mas eficaz para encontrar o público alvo proposto.

Quanto aos objetivos os 05 artigos observaram a coordenação motora das crianças com deficiência auditiva e o equilíbrio, associada a uma possível intervenção com atividade física para uma aquisição no desempenho motor da criança. E também houve uma preocupação dos autores em detalhar os métodos e os instrumentos para chegar até as analises dos resultados, o que facilitou a interpretação desses resultados para compor a presente análise.

Desses 05 artigos, 04 utilizaram como instrumento para avaliar o desempenho motor da criança com deficiência auditiva o teste de coordenação corporal KTK, composto por quatro tarefas: trave de equilíbrio, salto monopedal, salto lateral e transferência sobre a plataforma. O qual foi desenvolvido para diagnosticar deficiências motoras em crianças com lesões cerebrais e/ou desvios comportamentais. ${ }^{11}$

E apenas 01 artigo $^{13}$ utilizou um teste diferenciado dos demais o teste "do quatro" e de Romberg (equilíbrio estático), teste "passeio na trave" e de Unterberger (equilíbrio dinâmico), e giro de $180^{\circ}$ (equilíbrio recuperado) para avaliar o equilíbrio da criança.

Cabe destacar que maioria dos estudos aqui revisados, 04 de 05 artigos, realizaram intervenções com atividades motoras ligadas à Educação Física, nos quais o teste de coordenação corporal KTK foi utilizado para comparar resultados do pós-teste em relação aos resultados iniciais vistos no pré-teste. 
Os procedimentos adotados por Strapasson et al. ${ }^{18}$ seguiram uma intervenção baseada em dinâmicas corporais em aulas de Educação Física durante 4 meses, com 20 aulas no total de 60 minutos cada, na própria instituição que a criança se encontrava. Já Souza et al. ${ }^{6}$ adotaram uma intervenção de 14 sessões, baseada nas principais dificuldades apresentadas pelos sujeitos de pesquisa na avaliação do pré-teste, sendo um programa de intervenção especifico para minimizar as limitações motoras do grupo.

Por outro lado, Montezuma et al. ${ }^{8}$ aplicaram uma intervenção motora durante 4 meses com aulas de dança, totalizando 12 aulas de 50 minutos cada. E por fim, percebe-se que apenas no estudo de Pena e Gorla ${ }^{7}$ houve a preocupação dos pesquisadores em alocarem, além do grupo experimental, um grupo controle para o estudo. No estudo foram aplicadas 12 aulas de basquetebol, com duração de 60 minutos cada aula, de 3 a 4 vezes na semana.

Diferente dos demais estudos, Azevedo et al. ${ }^{13}$ utilizaram outro teste para avaliar o equilíbrio de pessoas com deficiência auditiva e de pessoas ouvintes, fazendo a comparação entre esses dois grupos, no período de 2 dias, sendo que cada dia em uma instituição, dessa maneira utilizou-se os seguintes testes: teste "do quatro" e de Romberg (equilíbrio estático), teste "passeio na trave" e de Unterberger (equilíbrio dinâmico), e giro de $180^{\circ}$ (equilíbrio recuperado). Dessa forma, observou-se nesse estudo, que além do teste ser diferente dos demais, os autores realizaram apenas a avaliação do equilíbrio e que não houve intervenção, sendo o estudo meramente de caracterização.

Sendo assim, nota-se que existem diversos instrumentos e testes para avaliar a coordenação motora de crianças com deficiência, mas através dos achados dos artigos analisados, observou-se que o KTK foi o teste mais utilizado, justificado pelo fato do KTK ser um teste validado, e bastante utilizado em diversos estudos, inclusive internacionais ${ }^{21-24}$ mostrando eficiência, principalmente quando aplicado de forma correta, além da construção dos instrumentos serem de baixo custo e de fácil aplicação.

Os 04 artigos que utilizaram o teste de $\mathrm{KTK}^{6-8,}, 18$ como foi relatado anteriormente, realizaram pré e pós-testes, sendo que, as crianças passaram por um período de intervenção Conexões: revista da Faculdade de Educação Física da UNICAMP, Campinas, v. 13, n. 3, p. 114-130, jul./set. 2015. ISSN: 1983-9030 
com aulas de Educação Física (Arvorismo, Basquetebol, Dança e Atividades Recreativas). Através dessas intervenções observou-se que as crianças tiveram ganhos na aquisição motora, pois resultados significativos foram observados.

Em relação aos principais resultados encontrados, observou-se que nos 05 artigos as crianças com deficiência auditiva apresentaram comprometimento e perturbação no desempenho motor. Essas dificuldades podem ser ocasionadas porque a criança com deficiência pode ter comprometimento no equilíbrio que é fundamental para a coordenação motora. ${ }^{11}$

E durante a realização do teste do KTK, segundo dados de $03 \operatorname{artigos}^{6-8}$ as atividades que as crianças tiveram mais dificuldade foi à trave de equilíbrio e saltos monopedais. Nota-se que essas duas atividades exigem que o sujeito tenha bastante atenção, pois além do equilíbrio, a criança precisa executar a marcha para trás sobre a trave, e também ter uma coordenação dos membros inferiores, a qual exige força. Sendo assim, Montezuma et al. ${ }^{8}$ apontam que essa piora nesses testes deve ser explicada porque as crianças possuem um repertório motor pobre, que durante a infância não foi estimulado. Enquanto no teste de salto lateral e transferência sobre a plataforma, que exige velocidade em saltos alternados e organização espacial, as crianças tiveram resultados significativos. ${ }^{6,8}$

Dessa maneira, percebe-se a importância das aulas de Educação Física na infância, pois é através da mesma, que a criança é estimulada a desenvolver as habilidades motoras básicas, fazendo com que a criança tenha um repertório motor rico. E quando se fala em crianças com deficiência auditiva quanto mais cedo essas intervenções forem feitas, melhores serão os resultados no desempenho motor da criança.

Os autores dos artigos aqui revisados apontaram como limitações dos estudos alguns fatores externos que possam ter influenciado os resultados da pesquisa, sendo um desses fatores o tamanho da amostra, tendo como maior amostra dos artigos selecionados o de Souza et al. ${ }^{6}$ com 23 indivíduos, que apesar de terem existido alguns fatores externos e 
individuais, como falta de concentração, atenção, impaciência entre outros, não influenciou nos resultados.

E como menor amostra o de Montezuma et al. ${ }^{8}$ com apenas 05 sujeitos, pois como se tratava de uma intervenção com Dança os autores preferiram intervir com número reduzido de sujeitos. Apesar da discrepância do tamanho da amostra evidenciada nos estudos de Souza et al. ${ }^{6}$ em relação ao de Montezuma et al., ${ }^{8}$ observou-se que independente do tamanho da amostra, nos 02 artigos os indivíduos com surdez tiveram resultados positivos no desempenho motor.

Outro fator citado pelos autores que limitou o estudo foi um curto período de intervenções, pois, os autores acreditam que um período maior de intervenção traria melhores resultados na aquisição motora da criança, visto que uma intervenção de 3 a 4 meses seria adequada, já que houve artigo com intervenção de apenas uma semana.

Os estudos de Pena e Gorla, ${ }^{7}$ Montezuma et al. $^{8}$ e Strapasson et al. ${ }^{18}$ mostram a importância da atividade física como o Basquetebol, Dança e atividades recreativas na melhoria da coordenação motora da criança, como na comunicação, ritmo e participação. Pois, Pena e Gorla ${ }^{7}$ apontam que essa melhoria pós-teste, pode ter acontecido porque essas atividades físicas exigem muito controle corporal, como paradas bruscas, saídas rápidas, mudança de direção, estimulando assim que a criança tenha um ganho no desempenho corporal.

No estudo de Azevedo et al. ${ }^{13}$ em que foi realizado a comparação do grupo dos ouvintes e dos surdos, observou-se que os ouvintes apresentaram melhores resultados no equilíbrio dinâmico, estático e recuperado do que o grupo dos surdos. Os autores ainda falam que esse fato pode está relacionado com perda auditiva neurosensorial, que afeta o equilíbrio. Então uma criança tendo o equilíbrio comprometido poderá ter a coordenação motora afetada, fazendo com que a criança tenha dificuldade na realização nos testes aplicados. 
E por fim, estudos como o de Souza et al., ${ }^{6}$ Pena e Gorla, ${ }^{7}$ Montezuma et al., ${ }^{8}$ Strapasson et al. ${ }^{18}$ apontam a importância de um programa de Educação Física bem estruturado para o desenvolvimento da coordenação motora da criança com deficiência auditiva e como por meio de um período de intervenção específica poderão haver melhoras nas habilidades motoras. Uma vez que a crianças com deficiência auditiva apresentam dificuldades nos níveis de coordenação, ritmo e equilíbrio. Sendo assim, Gorla ${ }^{25}$ afirma que quanto mais cedo realizar o diagnóstico e a intervenção, melhor será o desenvolvimento da criança.

Pois, através desses achados observa-se o quanto um programa de atividade física estruturado é essencial para o desenvolvimento da coordenação motora de crianças e adolescentes. E principalmente quando estiver associada a algum teste que avalie o desempenho motor da criança desde cedo, facilitando assim a intervenção do profissional de Educação Física. Uma vez que a criança e/ou adolescente tenha o diagnóstico da coordenação motora, o professor procurará intervir para que os mesmos tenham ganhos nas habilidades motoras.

\section{CONCLUSÃO}

A partir dessa revisão sistemática, evidencia-se que ainda são realizados e publicados poucos estudos sobre avaliação da coordenação corporal em crianças e adolescentes com deficiência auditiva no Brasil. Contudo, evidenciou-se que os pesquisadores nacionais utilizam instrumentos fidedignos em suas avaliações motoras, o que demonstra que as pesquisas nessa área seguem procedimentos importantes no que tange a caraterização motora de um grupo populacional ainda pouco explorado em pesquisas.

A revisão demonstrou a importância de estudos que objetivaram avaliar efeitos de intervenção nesse público, tendo o instrumento KTK como um forte indicativo para avaliação de efeitos pré e pós-treinamento, mesmo sem ter localizado estudos de validação do instrumento para tal população no Brasil. 


\section{REFERÊNCIAS}

${ }^{1}$ GORGATTI, M. G., DA COSTA, R. F. Atividade física adaptada: qualidade de vida para pessoas com necessidades especiais. 2. ed. São Paulo: Manole, 2008.

${ }^{2}$ ROSSI, T. R. F. Brincar: uma opção para vencer o obstáculo da interação entre mãe ouvinte / filho surdo. 2000. Tese (Doutorado) - Universidade Estadual de Campinas, Faculdade de Educação Física, Campinas, 2000.

${ }^{3}$ TODD, N. W. At-risk populations for hearing impairment in infants and young children. International Journal of Pediatric Otorhinolaryngology, v. 29, n.1, p.11-21, mar. 1994. Disponível em: <http://www.ncbi.nlm.nih.gov/pubmed/8169043>. Acesso em: fev. 2014.

${ }^{4}$ MONTEIRO, C. F. S. et al. Suspeita da perda auditiva por familiares. Revista CEFAC: speech, language, hearing sciences and education journal, São Paulo, v. 11, n.3, p. 486493, 2009. Disponível em:< http://www.scielo.br/ >. Acesso em: jan. 2014.

${ }^{5}$ DANTAS, M. B. S. et al. Resultados de um programa de triagem auditiva neonatal em Maceió. Revista Brasileira de Otorrinolaringologia, São Paulo, v. 75, n. 1, p. 58-63, jan./fev. 2009. Disponível em: <http://www.scielo.br>. Acesso em: mar. 2014.

${ }^{6}$ SOUZA, A. N. et al. Análise da coordenação motora de pessoas surdas. Arquivos Ciência Saúde Unipar, Umuarama, v. 12, n. 3, p. 205-211, set./dez. 2008. Disponível em:<http://revistas.unipar.br/saude/>. Acesso em: fev. 2014.

${ }^{7}$ PENA, L. G. S.; GORLA, J. I. Coordenação motora em crianças com deficiência auditiva: avaliação e intervenção. CONEXÕES: revista da Faculdade de Educação Física da UNICAMP, Campinas, v. 8, n. 3, p. 104-123, set./dez. 2010. Disponível em:<http://www. http:// www.bibliotecadigital.unicamp.br>. Acesso em: jan. 2014. 
${ }^{8}$ MONTEZUMA, M. A. L. et al. Adolescentes com deficiência auditiva: a aprendizagem da dança e a coordenação motora. Revista Brasileira de Educação Especial, Marília, v.17, n. 2, p. 321-334, maio/ago. 2011. Disponível em:< http://www.scielo.br/ >. Acesso em: jan. 2014.

${ }^{9}$ PELlEGRINI, A. N. et al. Desenvolvendo a coordenação motora no ensino fundamental. São Paulo: Ed. da UNESP, 2005. Disponível em:<http://www.unesp.br/prograd/>. Acesso em: fev. 2014.

${ }^{10}$ KIPHARD, E. J.; SCHILLING, F. Körperkoordinationstest für kinder, KTK. Weinheim: Beltz. 1974.

${ }^{11}$ GORLA, J. I.; ARAÚJO, P. F.; RODRIGUES, J. L. Avaliação motora em educação física adaptada. São Paulo: Phorte. 2009.

${ }^{12}$ TUBINO, M. J. G. Metodologia do treinamento desportivo. São Paulo: IBRASA, 1979.

${ }^{13}$ AZEVEDO, M. G.; SAMELLI, A. G. Estudo comparativo do equilíbrio de crianças surdas e ouvintes. Revista CEFAC: speech, language, hearing sciences and education journal, São Paulo, v. 11, Supl. 1, p. 85-91, mar. 2009. Disponível em: <http://www.scielo.br>. Acesso em: jan. 2014.

${ }^{14}$ MOR, R.; TAGUCHI, K. C.; FIGUEIREDO, J. F. F. R. Vestibulometria e fonoaudiologia: como realizar e interpretar. São Paulo: Lovise; 2001.

${ }^{15}$ SCHMIDT, S. Hearing impaired students in physical education. Adapted Physical Activity Quarterly, Dallas, v. 2, n. 4, p. 300-306, oct. 1995. 
${ }^{16}$ NEGRINE, Airton. Educação psicomotora: a lateralidade e a orientação espacial. Porto Alegre: Pallotti, 1986.

${ }^{17}$ DANTAS, E.H.M. A prática da preparação física. 5. ed. Rio de Janeiro: Shape, 2003.

${ }^{18}$ STRAPASSON, A.M. et al. Teste ktk na avaliação da coordenação motora de pessoas com deficiência auditiva. Coleção Pesquisa em Educação Física, São Paulo, v. 8, n. 2, 177-182, fev. 2009. Disponível em:<http://www.fontouraeditora.com.br//>. Acesso em: jan. 2014.

${ }^{19}$ SILVA, R. P. Análise do nível de desenvolvimento motor de crianças surdas que praticam natação escolar. Brasília-DF. UniCEUB, 2013. Disponível em<:http://repositorio.uniceub.br/handle/235/3920>. Acesso em: Mar. 2014.

${ }^{20}$ RODRIGUES, C. G. Educação Física infantil: motricidade de 1 a 6 anos. São Paulo: Phorte, 2005.

${ }^{21}$ GHEYSEN, F.; LOOTS, G.; WAEVELDE, H. V. Motor development of deaf children with and without cochlear implants. Journal of Deaf Studies and Deaf Education, Oxford, v. 13, n. 2, p. 215-224, apr. 2008.

${ }^{22}$ VANDORPE, B. et al. The körperkoordinations test für kinder: reference values and suitability for 6-12-year-old children in Flanders. Scandinavian Journal of Medicine and Science in Sports, v. 21, n. 3, p. 378-388, jun. 2011.

${ }^{23}$ TOFTEGAARD-STOECKEL, J.; GROENFELDT, V.; ANDERSEN, L. B. Children's self-perceived bodily competencies and associations with motor skills, body mass index, teachers' evaluations, and parents' concerns. Journal of Sports Sciences, v. 28, n.12, 2010, p.1369-1375, 2010. 
${ }^{24}$ VANDENDRIESSCHE, J. B. et al. Multivariate association among morphology, fitness, and motor coordination characteristics in boys age 7 to 11. Pediatric Exercise Science, v. 23, n. 4, p. 504-520, nov. 2011.

${ }^{25}$ GORLA, J. I. Coordenação corporal de portadores de deficiência mental: avaliação e intervenção. 2001. 134f. Dissertação (Mestrado em Educação Física) - Universidade Estadual de Campinas. Faculdade de Educação Física, Campinas, 2001.

Recebido em: 04 abr. 2014

Aceito em: 02 set. 2015

Contato: jorgelcneto@hotmail.com 